\title{
Evaluation of an influenza-like illness case definition in the diagnosis of influenza among patients with acute febrile illness in cambodia
}

\author{
Matthew R Kasper ${ }^{1 *}$, Thomas F Wierzba², Ly Sovann³, Patrick J Blair ${ }^{1}$, Shannon D Putnam ${ }^{1}$
}

\begin{abstract}
Background: Influenza-like illness (ILI) is often defined as fever $\left(>38.0^{\circ} \mathrm{C}\right)$ with cough or sore throat. In this study, we tested the sensitivity, specificity, and positive and negative predictive values of this case definition in a Cambodia patient population.

Methods: Passive clinic-based surveillance was established at nine healthcare centers to identify the causes of acute undifferentiated fever in patients aged two years and older seeking treatment. Fever was defined as tympanic membrane temperature $>38^{\circ} \mathrm{C}$ lasting more than 24 hours and less than 10 days. Influenza virus infections were identified by polymerase chain reaction.

Results: From July 2008 to December 2008, 2,639 patients were enrolled. From 884 (33\%) patients positive for influenza, 652 presented with ILI and 232 acute fever patients presented without ILI. Analysis by age group identified no significant differences between influenza positive patients from the two groups. Positive predictive values (PPVs) varied during the course of the influenza season and among age groups.

Conclusion: The ILI case definition can be used to identify a significant percentage of patients with influenza infection during the influenza season in Cambodia, assisting healthcare providers in its diagnosis and treatment. However, testing samples based on the criteria of fever alone increased our case detection by $34 \%$.
\end{abstract}

\section{Background}

Globally, influenza is considered one of the most important infectious diseases. It is reported that between 3 and 5 million cases of severe influenza disease occur each year [1], with estimated annual influenza-associated mortality between 500,000 and 1,000,000 cases (median case-fatality of 190 deaths per 100,000 person infected with influenza) $[2,3]$. Complicating the global influenza burden is the recent recognition of a novel quadreassortment swine-origin influenza $\mathrm{A}$ virus which is the agent associated with the WHO declared influenza pandemic [4].

Influenza viruses are transmitted through the respiratory route [5-8] and infections vary from asymptomatic to severe, life threatening. Common clinical symptoms of influenza include fever, cough, sore throat, headache,

\footnotetext{
* Correspondence: kaspernamru2@yahoo.com

'U.S. Naval Medical Research Unit 2, Jakarta, Indonesia

Full list of author information is available at the end of the article
}

muscle aches, nasal congestion and weakness [9]. These symptoms can be non-specific and do not easily distinguish influenza from other respiratory viral syndromes or other infectious etiologies in patients presenting for healthcare services with acute febrile illness [10]. As with any standardized syndromic disease case definition, definitions of "influenza-like illness" (ILI) vary [11-13] but typically include fever $\left(\geq 38^{\circ} \mathrm{C}\right)$ with one or more respiratory symptoms (e.g., cough or sore throat). These clinical algorithms have been studied in children and adults as part of hospital-based or age-specific antiviral trials. These studies suggest that an ILI definition including cough has a positive predictive value (PPV) of $60 \%$ to $87 \%[11,14,15]$. Other work has focused on influenza in hospitalized patients $[16,17]$ and in national surveillance activities [18]. The majority of these efforts have focused on populations from developed countries, whereas information on the predictive values of ILI symptoms in developing countries is limited. 
Building upon previous influenza surveillance results from rural Cambodian patients [19], we evaluated a commonly used ILI definition, document fever and cough or sore throat as a predictor of influenza disease. As previous studies have suggested limiting the use of clinical predictors for influenza to the influenza season [15], the sampling included all eligible patients enrolled during a single Cambodian influenza season.

\section{Methods}

\section{Study site and population}

In December 2006, a clinic and hospital-based acute febrile illness surveillance was implemented at nine Cambodian government medical clinics. Five of these sites were located in Operational District A (peri-urban) and four were in Operational District B (rural). All participating field sites were within 50 kilometers of Phnom Penh in south-central Cambodia. Patients were recruited by study site staff if they had a recorded temperature $\geq$ $38.0^{\circ} \mathrm{C}$ lasting at least 24 hours but not greater than 10 days, were two years of age or older, and, after medical examination, had no obvious source of infection. A healthcare provider in each clinic obtained written informed consent, administered a pre-tested enrollment questionnaire, performed a medical examination and collected clinical specimens per study protocol[19]. Influenza-like illness was defined according the WHO guidelines, which included, documented fever $\left(\geq 38.0^{\circ} \mathrm{C}\right)$ and cough or sore throat. For this study, only patients enrolled I the surveillance for acute febrile illness from July 2008 through December 2008 were included for analysis; corresponding to influenza season in Cambodia.

\section{Specimen Collection}

For each enrolled patient, one throat and one nasal swab were collected. For nasal swabs, a dry polyester swab was inserted into the nostril parallel to the palate, slowly withdrawn, and placed in a vial containing $2-3$ milliliters of virus transport medium (VTM). For throat swabs, both tonsils and the posterior pharynx were swabbed vigorously, and the swab placed in $2-3$ milliliters of VTM. All inoculated vials were kept at $4{ }^{\circ} \mathrm{C}$ until transported between 24 and 72 hours after collection to the Naval Medical Research Unit No. 2 (NAMRU-2) located at the campus of the Cambodian National Institute of Public Health (NIPH).

\section{Laboratory testing}

Ribonucleic acid (RNA) was extracted from nasal and throat swabs using QIAamp viral RNA mini kits (QIAGEN, Hilden, Germany) following the manufacturer's instructions and stored at $-70^{\circ} \mathrm{C}$. The influenza virus genome was detected using a reverse real-time PCR
(rRT-PCR) assay developed to detect influenza A/H1, $\mathrm{A} / \mathrm{H} 3, \mathrm{~A} / \mathrm{H} 5$, and $\mathrm{B}$ virus subtypes. Real-time assays were developed at the Centers for Disease Control and Prevention (Atlanta, GA, USA). Real time assays and sequence information are available upon request from Dr. Steve Lindstrom under the terms of a material transfer agreement. One-step rRT-PCR was performed in a final volume of $25 \mu \mathrm{l}$ containing $5 \mu \mathrm{l}$ of extracted RNA, $12.5 \mu \mathrm{l}$ of buffer mix and $0.5 \mu$ l Superscript III/ Platinum Taq-Enzyme mix, 20 units of RNAse-out (Invitrogen, Carlsbad, CA, USA), $0.8 \mu \mathrm{M}$ for each primer and $0.2 \mu \mathrm{M}$ of probe. The Rotor-Gene 6000 real-time thermocyler (Corbett Life Science, Sydney, Australia) was used for all PCR reactions. The thermocycling parameters for all targets consisted of $50^{\circ} \mathrm{C}$ for 30 minutes, $95^{\circ} \mathrm{C}$ for 2 minutes, and 45 cycles with $95^{\circ} \mathrm{C}$ for 15 seconds, $55^{\circ} \mathrm{C}$ for 30 seconds (US CDC, Atlanta, GA, USA).

\section{Statistical Analysis}

All data was double-data entered into MS Access (Microsoft Inc., Redmond, WA, USA). Data was imported into SAS v9.1 (SAS, Cary, NC), which was used for all statistical analyses. The definition of ILI (fever and cough or sore throat) was analyzed to determine sensitivity, specificity, positive predictive valve (PPV) and negative predictive value (NPV) comparing patients with and without laboratory evidence of influenza virus. Sensitivity was defined as the probability of having the ILI given laboratory-confirmed influenza; specificity was defined as the probability of not having the ILI when the patient does not have laboratory-confirmed influenza. The PPV was the probability of having laboratory-confirmed influenza when ILI was present; NPV is the probability of not having laboratoryconfirmed influenza when ILI was not present[15]. Categorical data was analyzed by the use of either ChiSquare (expected cell frequency $>5$ ) or Fisher's Exact Test (expected cell frequency $\leq 5$ ). Continuous data were assessed for normality and if normally distributed, parametric statistics were used. If the data was non-normally distributed, then non-parametric testing was used. All statistical tests were two-tailed and significance was defined as $\mathrm{p}<0.05$.

\section{Ethical Considerations}

Eligible subjects were voluntarily enrolled in accordance with an Institutional Review Board protocol approved by U.S. NAMRU-2 and the National Ethics Committee of the Royal Kingdom of Cambodia, Ministry of Health.

\section{Results}

From July 2008 through December 2008, a total of 2,639 febrile patients were enrolled. The median age of 
enrolled patients was 11 years (Interquartile Range (IQR) 6 - 25) and males accounted for $52.9 \%$ of enrolled patients. The median day of patient presentation was at 3 days of fever duration (IQR $3-4$ ) with a median temperature of 39.0 (IQR 38.5 - 39.5). A more detailed description of this population has been described elsewhere [19].

Among the enrolled patients, the prevalence of laboratory-confirmed influenza was $33.5 \%$ (884) and the proportion of patients with ILI was $62.2 \%(1,652)$. Based on these values, the sensitivity and specificity of the ILI cases definition to predict disease was $73.8 \%(652 / 884)$ and $43.0 \%$ (755/1755), respectively. The PPV was $39.5 \%$ (652/1652) and NPV was 76.5\% (755/987). Among enrolled patients, laboratory testing of patients using the criteria of only fever increased the number of detected cases from 652 to 884 , a $34 \%$ increase.

Of the 884 laboratory-confirmed influenza cases, $69 \%$ (608) and 31\% (276) had influenza A and B types, respectively. (Table 1) Among patients with influenza A and B, ILI sensitivity was $76.0 \%(462 / 608)$ and $68 \%$ (190/276), respectively suggesting that the ILI case definition was 1.4 times (95\% confidence interval $(\mathrm{CI}): 1.03$ to $1.99, \mathrm{p}=0.025)$ more sensitivity in detecting influenza A as compared to than influenza B cases. Sensitivity did not differ between influenza $\mathrm{H} 3$ and $\mathrm{H} 1$ types.

When stratified by age groups (young children (ages $2-6)$, school aged children (ages 7-18) and adults ( $>18$ years of age)), there was no detected statistically significant difference for ILI sensitivity. However, there were noted statistically significant differences by age group for specificity ( $\mathrm{p}<.0001)$, PPV ( $\mathrm{p}=.01)$, and NPV $(\mathrm{p}<.0001)$. (Table 2) For all three values, analysis suggested that adults had higher specificity (53\%), NPV (90\%), and lower PPV (21\%) as compared to subjects in the younger age groups.

Table 1 Patients ( $N=2,639)$ presenting with influenzalike illness and laboratory confirmed Influenza type and subtype by clinical presentation of Influenza-like IIIness (ILI)

\begin{tabular}{cccc}
\hline Influenza Status & ILI & NO ILI & Total \\
\hline Influenza (overall) & $652(73.8 \%)^{*}$ & 232 & 884 \\
$\mathrm{~A}+$ & $462(76.0 \%)$ & 146 & 608 \\
$\mathrm{~A} / \mathrm{H} 1 \neq$ & $78(74.2 \%)$ & 27 & 105 \\
$\mathrm{~A} / \mathrm{H3}$ & $383(76.3 \%)$ & 119 & 502 \\
$\mathrm{~A} / \mathrm{H} 5$ & $1(100 \%)$ & 0 & 1 \\
$\mathrm{~B}$ & $190(68.8 \%)$ & 86 & 276 \\
Negative & 1000 & 755 & 1,755
\end{tabular}

* $\mathrm{n}$ (\% positive for ILI).

† Odds ratio $=1.4(95 \%$ confidence internals: 1.03 to $1.99, p=0.025)$ for frequency of ILI by influenza A (76.0\%) and influenza B $(68.8 \%)$ types.

‡ ILI for $\mathrm{H} 1$ (74.2\%) vs. H3 (76.3\%), $\mathrm{p}=.66$.
Table 2 Sensitivity, Specificity, positive and negative predictive value for a case definition of Influenza-like Illness by presence or absence of laboratory-confirmed influenza virus infection

\begin{tabular}{lllll}
\hline Age (years) & $\mathbf{2}$ to $\mathbf{6}$ & $\mathbf{7}$ to $\mathbf{1 8}$ & $\mathbf{> 1 8}$ & p-value \\
\hline Sensitivity & $76(335)^{*}$ & $73(401)$ & $68(148)$ & .18 \\
Specificity & $32(470)$ & $37(487)$ & $53(798)$ & $<.0001$ \\
Positive Predictive Value & $44(576)$ & $49(599)$ & $21(476)$ & .01 \\
Negative Predictive Value & $66(229)$ & $63(289)$ & $90(471)$ & $<.0001$ \\
\hline
\end{tabular}

* Percent (number tested).

When stratified by month, the PPV was lowest in July for all age groups, peaking in October, and declined thereafter (Table 3 ). While the monthly pattern was the same for all, the PPV statistically differed by age group for September through December. PPVs during these months were lowest for adults, although 2 to 5 years olds had lower rates than 7 to 18 year olds in December. Over the entire period of study the PPVs for age groups 2 to 6,7 to 18 , and $>18$ years was $44 \%, 49 \%$ and $21 \%(\mathrm{p}<.0001)$, respectively. Again, adults had the lowest overall PPV.

From July to December 2008, ILI was documented in $43 \%$ to $68 \%$ of enrolled acute fever patients (Figure 1) per month while the prevalence of influenza ranged from $9.9 \%$ in July to a peak of $59.4 \%$ in October. ILI criteria was met most often in December (68\%), when the prevalence of influenza was only $9.7 \%$.

\section{Discussion}

Previous studies have reviewed the definition of ILI and symptoms most commonly associated with influenza disease, with most studies using hospitalized patients [9,11-13]. This analysis evaluated the ability of an ILIdefined syndrome to correctly identify patients with a laboratory-confirmed influenza infection in Cambodia patients seeking treatment for acute febrile illness. We found that the ILI case definition was more sensitive for patients infected with influenza A than influenza B, possibly reflecting milder disease among those infected influenza $B$ as described in previous studies $[20,21]$.

Table 3 Positive predictive value of having influenza virus given ILI symptoms stratified by age group and enrollment month

\begin{tabular}{ccccc}
\hline Age (years) & $\mathbf{2 - 6}$ & $\mathbf{7 - 1 8}$ & $\mathbf{> 1 8}$ & p-value \\
\hline July & 4 & 16 & 9 & .10 \\
August & 22 & 24 & 19 & .78 \\
September & 60 & 59 & 26 & $<.0001$ \\
October & 65 & 73 & 33 & $<.0001$ \\
November & 53 & 52 & 20 & $<.001$ \\
December & 9 & 32 & 7 & $<.0001$ \\
OVERALL & 44 & 49 & 21 & $<.0001$ \\
\hline
\end{tabular}




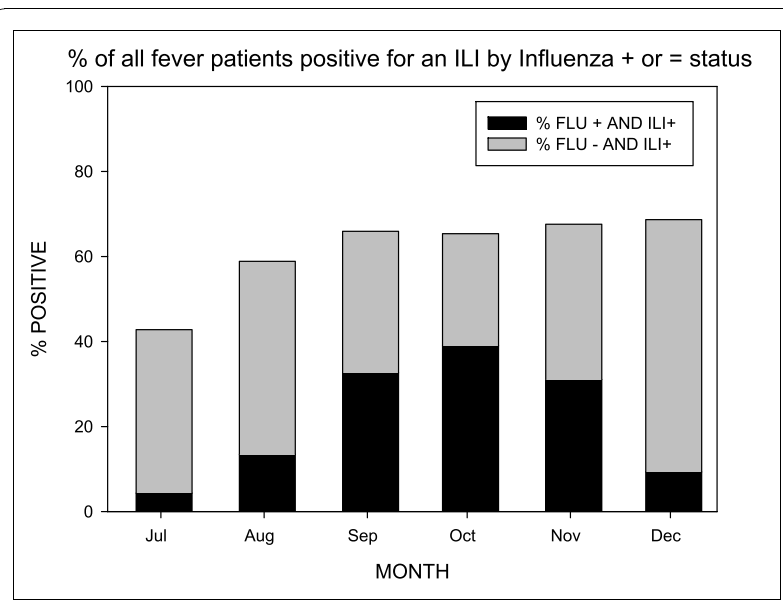

Figure 1 Among patients with an acute febrile illness, the percentage of patients with ILI that were positive or negative for influenza infection.

The PPV of the ILI definition changed by month as changes in the prevalence of influenza infections occurred. Hence the PPV appeared to peak in October, at the peak of the influenza season. The sensitivity of ILI case definition did not appear to vary with age. However, the specificity appeared highest in adults relative to children suggesting that adults were less likely to present with cough or sore throat than children possibly due to acquired immunity to respiratory agents that might induce ILI symptoms.

Laboratory testing patients only with ILI as enrollment criteria would have identified $74 \%$ of the influenza cases among enrolled patients. The definition of ILI for surveillance purposes is sufficient as a testing strategy to monitor seasonal influenza circulation and emerging influenza strains. The ILI definition did not detect $26 \%$ of cases in our clinic-based surveillance and may be lacking for clinical practice and treatment guidelines without laboratory testing to support the diagnosis.

The study enrollment criteria of reported fever duration for at least 24 hours does differ from the WHO ILI definition that has no defined duration of fever. It is possible that few patients seeking health care very early in their illness may not meet study enrollment criteria and therefore not been enrolled. However, the median day of fever reported among patients upon enrollment was three days with an IQR of 3-4 days, suggesting that most Cambodian patients were unlikely to seek care until well into their disease. In addition, further work is needed to understand the healthcare seeking behavior of this study population.

From August 2008-December 2008, the percentage of febrile patients with ILI stayed constant study despite a varying prevalence of influenza, suggesting that other pathogens are contributing to febrile respiratory illness in Cambodia. In addition to influenza virus, recent studies have identified other respiratory pathogens such as human metapneumovirus [22], respiratory syncytial virus, and parainfluenza virus 3 [23], adenovirus and rhinovirus from patients in developing countries; Further studies are required to determine the contribution of non-influenza respiratory pathogens among Cambodians presenting with ILI. The pathogens endemic (e.g. dengue virus and malaria) to this region and the different age groups they are most commonly detected in must also be considered.

Previous studies have determined that the best use of predictors is during the influenza season [15]. However, these studies have focused on seasonal influenza in developed nations and compared to these studies, the PPV of ILI identified in Cambodia was lower, even at the peak period of seasonal transmission. The range of the monthly prevalence over the course of the influenza season had considerable impact on the predictive values and suggest that even within an influenza season the use of clinical predictors may have limitations.

\section{Conclusions}

This study allowed us the unique opportunity to evaluate an ILI definition among all patients with an acute fever regardless of associated symptoms. Based on our results, the ILI case definition can be used to identify a significant percentage of patients with influenza infection during the influenza season in Cambodia. However, testing samples based on the criteria of fever alone increased our case detection by $34 \%$. Surveillance for acute fever provided a significant increase in the number of influenza patients identified and should be considered when determining the prevalence of influenza in a population or when using clinical case definitions to study the transmission of influenza among populations or study groups. While the ILI definition may indeed identify the majority of cases in a population presenting for treatment, it may underestimate the true burden of influenza disease among persons in resource-poor regions.

Those patients without respiratory symptoms who nonetheless tested positive for influenza are a reflection of the difficulty of its clinical diagnosis, a difficulty compounded by pathogens endemic to the region presenting as acute undifferentiated febrile illness such as dengue, malaria, typhoid fever, and other respiratory pathogens.

\section{Acknowledgements}

The authors are grateful to the clinicians and staff at the field sites in Cambodia for their assistance in enrolling and sampling patients. We thank the laboratories at NAMRU-2-Phnom Penh and the CDC for their work. We would like to thank Dr. Mark Riddle for his review of the manuscript. The views expressed herein are those of the authors and do not represent those 
of the US Department of Health and Human Services, the Department of Defense or the Department of the Navy.

Funding for this study was provided by the Global Emerging Infections Surveillance and Response System, a Division of the Armed Forces Health Surveillance Center; the U.S. Center for Disease Control and Prevention; and the Biosecurity Engagement Program, U.S. State Department.

\section{Author details}

${ }^{1}$ U.S. Naval Medical Research Unit 2, Jakarta, Indonesia. ${ }^{2}$ U.S. Naval Medical Research Unit No. 2, Phnom Penh, Cambodia. ${ }^{3}$ Communicable Diseases Control Department, Kingdom of Cambodia Ministry of Health, Phnom Penh, Kingdom of Cambodia.

\section{Authors' contributions}

MRK - Data analysis, writing manuscript. TFW - Data analysis, writing manuscript. LS - Review of manuscript. PJB - Study design, review and corrections of manuscript. SDP - study design, data analysis, writing manuscript. All authors read and approved the final manuscript.

\section{Competing interests}

The authors declare that they have no competing interests.

Received: 2 March 2010 Accepted: 7 November 2010 Published: 7 November 2010

\section{References}

1. Russell CA, Jones TC, Barr IG, Cox NJ, Garten RJ, Gregory V, Gust ID, Hampson AW, Hay AJ, Hurt AC, et al: The global circulation of seasonal influenza A (H3N2) viruses. In Science. Volume 320. New York, NY; 2008:(5874):340-346.

2. Nicholson KG, Wood JM, Zambon M: Influenza. Lancet 2003, 362(9397):1733-1745.

3. Stohr K: Influenza-WHO cares. Lancet Infect Dis 2002, 2(9):517.

4. Dawood FS, Jain S, Finelli L, Shaw MW, Lindstrom S, Garten RJ, Gubareva LV, Xu X, Bridges CB, Uyeki TM: Emergence of a novel swineorigin influenza $A(\mathrm{H} 1 \mathrm{~N} 1)$ virus in humans. The New England journal of medicine 2009, 360(25):2605-2615.

5. Dilantika C, Sedyaningsih ER, Kasper MR, Agtini M, Listiyaningish E, Uyeki TM, Burgess TH, Blair PJ, Putnam SD: Influenza virus infection among pediatric patients reporting diarrhea and influenza-like illness. $B M C$ infectious diseases 10(1):3.

6. Foy HM, Cooney MK, Allan ID, Albrecht JK: Influenza B in households: virus shedding without symptoms or antibody response. American journal of epidemiology 1987, 126(3):506-515.

7. Hayden FG, Fritz R, Lobo MC, Alvord W, Strober W, Straus SE: Local and systemic cytokine responses during experimental human influenza $A$ virus infection. Relation to symptom formation and host defense. The Journal of clinical investigation 1998, 101(3):643-649.

8. Stelzer-Braid S, Oliver BG, Blazey AJ, Argent E, Newsome TP, Rawlinson WD, Tovey ER: Exhalation of respiratory viruses by breathing, coughing, and talking. Journal of medical virology 2009, 81(9):1674-1679.

9. Cate TR: Clinical manifestations and consequences of influenza. The American journal of medicine 1987, 82(6A):15-19.

10. Call SA, Vollenweider MA, Hornung CA, Simel DL, McKinney WP: Does this patient have influenza? JAMA 2005, 293(8):987-997.

11. Boivin G, Hardy I, Tellier G, Maziade J: Predicting influenza infections during epidemics with use of a clinical case definition. Clin Infect Dis 2000, 31(5):1166-1169

12. Navarro-Mari JM, Perez-Ruiz M, Cantudo-Munoz P, Petit-Gancedo C, Jimenez-Valera M, Rosa-Fraile M: Influenza-like illness criteria were poorly related to laboratory-confirmed influenza in a sentinel surveillance study. Journal of clinical epidemiology 2005, 58(3):275-279.

13. Nicholson KG: Clinical features of influenza. Seminars in respiratory infections 1992, 7(1):26-37.

14. Monto AS, Gravenstein S, Elliott M, Colopy M, Schweinle J: Clinical signs and symptoms predicting influenza infection. Arch Intern Med 2000, 160(21):3243-3247.

15. Ohmit SE, Monto AS: Symptomatic predictors of influenza virus positivity in children during the influenza season. Clin Infect Dis 2006, 43(5):564-568.
16. Babcock HM, Merz LR, Dubberke ER, Fraser VJ: Case-control study of clinical features of influenza in hospitalized patients. Infect Control Hosp Epidemiol 2008, 29(10):921-926.

17. Babcock HM, Merz LR, Fraser VJ: Is influenza an influenza-like illness? Clinical presentation of influenza in hospitalized patients. Infect Control Hosp Epidemiol 2006, 27(3):266-270.

18. Thursky K, Cordova SP, Smith D, Kelly H: Working towards a simple case definition for influenza surveillance. J Clin Virol 2003, 27(2):170-179.

19. Blair PJ, Wierzba TF, Touch S, Vonthanak S, Xu X, Garten RJ, OkomoAdhiambo MA, Klimov Al, Kasper MR, Putnam SD: Influenza epidemiology and characterization of influenza viruses in patients seeking treatment for acute fever in Cambodia. Epidemiology and infection 2009, 1-11.

20. Proff R, Gershman K, Lezotte D, Nyquist AC: Case-based surveillance of influenza hospitalizations during 2004-2008, Colorado, USA. Emerging infectious diseases 2009, 15(6):892-898.

21. Thompson WW, Shay DK, Weintraub E, Brammer L, Bridges CB, Cox NJ, Fukuda K: Influenza-associated hospitalizations in the United States. Jama 2004, 292(11):1333-1340.

22. Abdullah Brooks W, Erdman D, Terebuh P, Klimov A, Goswami D, Sharmeen AT, Azim T, Luby S, Bridges C, Breiman R: Human metapneumovirus infection among children, Bangladesh. Emerging infectious diseases 2007, 13(10):1611-1613.

23. Broor S, Parveen S, Bharaj P, Prasad VS, Srinivasulu KN, Sumanth KM, Kapoor SK, Fowler K, Sullender WM: A prospective three-year cohort study of the epidemiology and virology of acute respiratory infections of children in rural India. PLoS One 2007, 2(6):e491.

\section{Pre-publication history}

The pre-publication history for this paper can be accessed here: http://www.biomedcentral.com/1471-2334/10/320/prepub

doi:10.1186/1471-2334-10-320

Cite this article as: Kasper et al:: Evaluation of an influenza-like illness case definition in the diagnosis of influenza among patients with acute febrile illness in cambodia. BMC Infectious Diseases 2010 10:320.

\section{Submit your next manuscript to BioMed Central and take full advantage of:}

- Convenient online submission

- Thorough peer review

- No space constraints or color figure charges

- Immediate publication on acceptance

- Inclusion in PubMed, CAS, Scopus and Google Scholar

- Research which is freely available for redistribution

Submit your manuscript at www.biomedcentral.com/submit
Ciomed Central 